CASE REPORTS

\title{
Phantom lymphadenopathy. An association with chronic fatigue syndrome
}

\section{D Shee}

Postgrad Med J 2003;79:59-60

Ten patients with self diagnosed enlarged lymph glands were referred to a general medicine outpatient clinic and careful examination did not confirm lymphadenopathy. All patients also complained of severe chronic fatigue associated with aches and miscellaneous somatic symptoms, and fulfilled criteria for diagnosis of chronic fatigue syndrome (CFS). Phantom lymphadenopathy may be a symptom in some people with CFS, and possible reasons for this are discussed.

T he situation where a patient complains of swollen glands but where a clinician cannot detect them appears to be common, but poorly described. From my personal disease registry I identified 10 patients (seven female) who have been seen in a hospital clinic over the last four years with "swollen glands" as a prominent symptom, but where physical examination in clinic failed to confirm lymphadenopathy. Case notes were examined retrospectively to see if there were any coexisting features in these patients.

\section{CASE REPORTS}

The 10 patients were referred to a general medicine clinic where the clinician does not have a specific interest in chronic fatigue syndrome (CFS). The primary reasons for referral mentioned in the general practitioners' letters were glandular enlargement (four patients), tiredness (two), sore throat, neck pain, chest pain, and wheeze (each one patient). Tiredness was mentioned somewhere in only five referral letters $(50 \%)$. Tiny lymph glands were mentioned as a finding in three referral letters. When seen in clinic all patients complained of swollen glands as one of their major symptoms. Patient details and sites of the alleged lymphadenopathy are shown in table 1 .

Nine of the 10 patients had previously been investigated by other specialists for medically unexplained symptoms (including variously: fatigue in four; abdominal pain, four; unexplained neurological symptoms, four; neck pain, three; headaches, two). Three patients had previously been treated for depression. All volunteered that they suffered from severe chronic fatigue and tiredness. Three patients had been fatigued for more than six months, four for over a year, and three for more than two years. Headache was common, and all described miscellaneous aches (table 1). Among other symptoms were paraesthesiae (five patients), low mood (five), sore throat (five), sleep disturbance (five), and sweats (four). Although lymphadenopathy was not detected in clinic, seven patients felt affected glands were swollen for most of the time. Some patients described variation in the size of the glands. Phrases used by different people included, "glands swell for a few hours each day", "stress swells the glands", and "glands worse in cold weather".

In all patients general and neurological examination was normal. Full blood count, erythrocyte sedimentation rate, renal function, electrolytes, thyroid and liver function tests were normal in all. Where performed, other investigations were also normal. Among others, these investigations included measurement of creatine phosphokinase (seven patients), serology for Epstein-Barr virus (five), and chest radiography (eight). Patients were reassured there was no evidence of serious pathology and advised gradually to increase daily activity. Review of case notes shows that in the brief period of follow up seven patients have subsequently been referred by their general practitioner to other clinics with further unexplained symptoms.

\section{DISCUSSION}

The patients fulfilled diagnostic criteria for CFS. ${ }^{12}$ All suffered from severe, disabling fatigue present for more than six months and associated with a combination of other qualifying symptoms such as myalgia, mood and sleep disturbance, and with no established medical condition known to produce fatigue. The female preponderance in CFS (seven of 10 patients) has previously been noted. ${ }^{2}$ Nine of 10 patients had previously been investigated in hospital clinics for assorted medically unexplained symptoms. Where investigations for these have been negative, patients are unlikely to have later evidence of serious underlying organic disease. ${ }^{3}$ The 10

\begin{tabular}{llllll|}
\hline Table 1 & \multicolumn{5}{l|}{ Patient characteristics } \\
\hline Patient No & Gender & Age (years) & Reported site of glands & Headache & Aches \\
\hline 1 & M & 23 & Cervical, groin & & Legs \\
2 & M & 32 & Cervical & + & Neck, ears, chest, arms \\
3 & $\mathrm{~F}$ & 50 & Cervical & + & Back, eyes, "muscles" \\
4 & $\mathrm{M}$ & 55 & Cervical, groin & + & Neck, back \\
5 & $\mathrm{~F}$ & 22 & Cervical, groin, axillae & + & Neck \\
6 & $\mathrm{~F}$ & 52 & Cervical & + & Neck, shoulders, thighs \\
7 & $\mathrm{~F}$ & 55 & Cervical & & Abdomen \\
8 & $\mathrm{~F}$ & 38 & Retroauricular & + & Neck, chest, breasts, back \\
9 & $\mathrm{~F}$ & 37 & Occipital & & Eyes, "muscles" \\
10 & $\mathrm{~F}$ & 43 & Cervical, occipital & + & Neck, chest, arms, back \\
\hline \multirow{2}{*}{ +, Symptom present. } & & & \\
\hline
\end{tabular}


patients had miscellaneous aches and a variety of other symptoms. A recent observational study suggests that substantial overlap in symptomatology exists across apparently diverse symptom syndromes such as fibromyalgia and CFS. ${ }^{4}$

The prevalence of true lymphadenopathy in CFS is uncertain. In one study palpable adenopathy was found in $17 \%$ of people with CFS, but also in $12 \%$ of controls with fatigue. ${ }^{5}$ An American paper mentions "tender cervical or axillary lymph nodes" as one of the possible qualifying symptoms for CFS, but with no reference to how often this is seen. ${ }^{6}$ However, the original British criteria for CFS state, "there are no clinical signs characteristic of the condition". An emerging consensus is that abnormal physical signs are conspicuously absent. ${ }^{2}$ The recent Department of Health working group report on CFS concluded that infections such as glandular fever could trigger $\mathrm{CFS}^{7}$ but that available evidence suggests that abnormal persistence of infectious agents does not occur in this condition. Similarly, the American criteria for CFS mention that apart from basic investigations no additional tests are recommended. ${ }^{6}$ The authors caution that serological tests for Epstein-Barr and other viruses do not confirm or exclude the diagnosis of CFS. ${ }^{6}$ Given the long histories and multiple symptoms and investigations in the 10 patients here described, it is unlikely their referral to me was triggered by an acute infection.

A large review of lymphadenopathy presenting in primary care concluded that it was common and in most cases no cause was found and it settled spontaneously. ${ }^{8}$ Only 3\% of patients were referred on for further investigation. ${ }^{8}$ The author does not mention if any patients presented with a complaint of swollen glands but without this being confirmed clinically. In an American internal medicine clinic fatigue was a presenting complaint in $8 \%$ of patients referred, but lymphadenopathy was not one of the 14 commonest presenting symptoms. ${ }^{9}$ I have been unable to find references to patients presenting with undetectable lymphadenopathy, although this entity must be seen periodically by most clinicians.

The patients genuinely felt they had swollen or inflamed lymph glands. Patients were not specifically asked about health checking behaviour such as feeling their neck for lymphadenopathy, but health anxiety is suggested by the number of specialists they had previously seen for medically unexplained symptoms. There is a large overlap in the diagnostic criteria for CFS and for fibromyalgia. ${ }^{4}$ Local areas of tenderness are common in both conditions, ${ }^{24}$ including tender spots around the neck and occipital region. Perhaps in some patients there is a misattribution with these tender areas being mistaken for painful lymph nodes? Similarly, somatisation is commonly associated with fatigue ${ }^{10}$ and discovery of transient small "shotty" glands (a common finding) could endow these with undue significance. Another explanation is that in some patients the glands may have been enlarged originally and subsequently settled, leaving an area of tenderness that is perceived as due to persisting or "phantom"
Learning points

- In primary care most lymphadenopathy is transient and rarely of significance.

- People with chronic fatigue syndrome often have multiple symptoms.

- Local areas of tenderness are common in chronic fatigue syndrome.

- The swollen glands mentioned by people with chronic fatigue syndrome are not always confirmed by the examining clinician.

glands. None of the patients had previously been labelled as having CFS so it is unlikely they had seen descriptions of tender lymph glands as a "sign" from information on the internet or available from self help organisations.

Review articles concerning causes of lymphadenopathy assume that it is actually present. I have been unable to find previous references to the situation where a patient complains of enlarged glands that the doctor is unable to feel. I am not describing a new syndrome but rather a symptom, that of phantom lymphadenopathy, which can be a prominent feature in some patients with CFS.

\section{Author's affiliation}

Department of Medicine, Queen Mary's Sidcup NHS Trust

Correspondence to: Dr C D Shee, Queen Mary's Hospital, Sidcup, Kent DA146LT, UK; charles.shee@qms-tr.sthames.nhs.uk

Submitted 27 May 2002

Accepted 5 November 2002

\section{REFERENCES}

1 Sharpe MC, Archard LC, Banatvala JE, et al. A report-chronic fatigue syndrome: guidelines for research. J R Soc Med 1991;84:1 18-21.

2 Wessely S, Hotopf $M$, Sharpe $M$, eds. CFS: definitions, epidemiology, presentation, prognosis. Chronic fatigue and its syndromes. Oxford: Oxford University Press, 1998: 141-57 (chapter 7).

3 Anonymous. What to do about medically unexplained symptoms [review]. Drugs and Therapeutics Bulletin 2001;39:5-8.

4 Wessely S, Nimnuan C, Sharpe M. Functional somatic syndromes: one or many? Lancet 1999;354:936-9.

5 Lane TJ, Manu P, Matthews DA. Depression and somatisation in the chronic fatigue syndrome. Am J Med 1991;91:335-44.

6 Fukuda K, Straus SE, Hickie I, et al. The chronic fatigue syndrome: a comprehensive approach to its definition and study. Ann Intern Med 1994:121:953-9.

7 Report of the CFS/ME Working Group. Report to the Chief Medical Officer of an independent working group. London: Department of Health, 2002.

8 Williamson HA. Lymphadenopathy in a family practice: a descriptive study of 249 cases. J Fam Pract 1985;20:449-52.

9 Kroenke K Mangelsdorff AD Common symptoms in ambulatory care: incidence, evaluation, therapy and outcome. Am J Med 1989;86:262-6.

10 Wessely $S$, Hotopf $M$, Sharpe $M$, eds. Fatigue and emotional disorders. Chronic fatigue and its syndromes. Oxford: Oxford University Press, 1998: 87-101 (chapter 4). 